\title{
L'italianistica brasiliana: alcune prospettive per la ricerca Tommaso Raso
}

\begin{abstract}
L'articolo valuta la situazione dell'italianistica brasiliana sotto il profilo della ricerca e offre alcuni spunti di riflessione per il futuro. Da una parte si valuta l'aspetto organizzativo degli studi, della ricerca e degli scambi con colleghi e con l'Iralia. Dall'altra si suggeriscono alcune aree di interesse primario per gli studi italianistici brasiliani. In particolare si mette l'accento sulla necessità di contribuire con oggerti di studio che approfondiscano le aree di contatto tra Brasile e Italia. Infine, l'arricolo fornisce le indicazioni dei principali strumenti bibliografici per l'italianista brasiliano.
\end{abstract}

PAROLE CHIAVE: italiano; ricerca; contatto.

L'italianistica brasiliana, riunitasi nell'ultimo congresso a Foz do Iguaçu nel giugno 2005, ha avviato una discussione sulle prospettive di ricerca e sui modi migliori per promuoverla ${ }^{1}$. Questo articolo vuole essere una continuazione di tale dibatrito, riprendendo gran parte delle considerazioni fatte in quell'occasione e aggiungendone di nuove. Lo scopo di questo articolo non è certo conclusivo; al contrario, esso nasce dalla convinzione che la comunita debba fare uno sforzo costante per strutturarsi scientificamente e che questo sforzo, incoraggiato dall'attuale presidenza e da alcuni fra i membri più autorevoli dell'Associaçāo Brasileira dos Professores de Italiano (ABPI), sia appena all'inizio. Naturalmente le prospettive della ricerca non riguardano solo i contenuti, ma anche il sostegno esterno di cui essa ha bisogno e le qualità organizzative che possono alimentarla. Per questa ragione, prima di

1. La discussione è avvenuta durante una tavola rotonda a cui hanno partecipato il prof. Mauro Porru (UFBA), presidente dell'ABPI, la prof.ssa Loredana Caprara (USP), il prof. Maurizio Babini (UNESP) e chi scrive (UFMG). 
parlare dei temi di ricerca che si offrono alla comunità degli italianisti brasiliani, in questo articolo si cerca di affrontare il più ampio argomento dell'organizzazione necessaria perché maturi meglio una comunità in grado di essere produttiva al di là dell'iniziativa lodevole dei singoli.

\section{La comunicazionc}

Una prima considerazione importante riguarda le difficoltà di comunicazione all'interno dell'italianistica brasiliana e tra questa e i ricercatori italiani; si tratta di una difficoltà di cui risente anche questo articolo. Il Brasile è un paese molto ampio, in cui necessariamente gli spostamenti sono difficili e costosi. Se a ciò si aggiunge la scarsità di fondi che le istituzioni universitarie brasiliane dedicano agli scambi fra docenti e studiosi di varie sedi, si ha un primo quadro delle difficoltà che questi hanno per incontrarsi, incontrare i ricercatori italiani e costruire progetti comuni. Ciò è vero per tutte le discipline, ma è ancor più vero per quelle discipline, tra cui la nostra, che hanno il loro centro di irradiazione fuori del paese e che non possono vantare una forza numerica ed economica come accade per le discipline che interessano lingue come inglese e spagnolo. LABPI organizza in media ogni due anni un congresso che costituisce la principale occasione di incontro fra i docenti e i ricercatori della disciplina. Tuttavia, per le difficoltà appena espresse, non tutti riescono a parteciparvi, soprattutto se l'incontro è organizzato in una sede lontana, e anche chi vi partecipa sente ovviamente la necessità di avere a disposizione altre occasioni. In una cornice siffatta, è vitale per i membri dell'italianistica la possibilità di affidarsi a scambi telematici che non siano soltanto quelli privati fra i membri che già si conoscono $\mathrm{e}$ in qualche modo già collaborano, ma uno spazio istituzionale, curato, se possibile, dall'ABPI. Se ne è parlato nell'ultimo convegno dove è stato anche presentato un sito. $\mathrm{Al}$ sito e ai suoi curatori dovrebbe perciò competere una importante funzione informativa e comunicativa verso e fra $\mathrm{i}$ soci.

Una buona comunicazione all'interno dell'italianistica brasiliana potrebbe permettere tra l'altro a tutti di venire tempestivamente a conoscenza della presenza di 
docenti italiani in Brasile. Periodicamente, infatti, una sede universitaria brasiliana riesce a ottenere i finanziamenti per invitare un ricercatore dall'Italia. Essere al corrente per tempo di queste rare occasioni sarebbe prezioso per le altre sedi che, con la semplice spesa di un biglietro interno e dell'alloggio, potrebbero beneficiarsi anch'esse della presenza dello studioso.

Una ulteriore caratteristica dell'ambiente accademico brasiliano, in particolare per le scienze umane, è la presenza di molte riviste che spesso non sono in condizioni di mantenere una periodicità fissa e di selezionare veramente i testi che pubblicano. $\mathrm{Ne}$ consegue che a una quantità di pubblicazioni talvolta anche eccessiva corrisponde la grande difficolrà, quando non l'impossibilità, di procurarsi i testi fuori dalle sedi di pubblicazione degli stessi e, non raramente, l'impossibilità di venire a conoscenza di cosa è stato pubblicato. Pertanto, molti lavori, nonostante siano stati pubblicati, rimangono del tutto ignoti alla comunità scientifica e non generano effettivamente conoscenza. La pubblicazione acquista cosi un valore autoreferenziale, funzionale quasi solo al curriculum del singolo ricercatore, e non uno strumento di conoscenza e di arricchimento per la comunità e per gli studi. Questo, come si è detro, non è certo una peculiarità dell'italianistica, ma è un problema comune a tutte le discipline umanistiche. Sarebbe senz'altro auspicabile che una o due di queste riviste acquisissero una visibilità almeno nazionale e fungessero da punto di riferimento per la comunità, attraendo il meglio dei lavori che si fanno in Brasile, ma anche dando notizia periodicamente di quanto accade che sia di interesse per la comunità. La presenza di una o due riviste chiaramente di spicco permetterebbe agli studiosi di tenersi costantemente al corrente delle più importanti novità e renderebbe possibile una rappresentatività effettiva degli studi svolti in Brasile anche fuori dal paese e soprattutto in Italia.

Analogamente, le pubblicazioni su rivista e in libro che interessino l'italianistica brasiliana spesso sono di difficile reperimento. Sarebbe utilissimo eleggere una sede più agevole da raggiungere, come ad esempio $S$. Paulo, e costituirvi presso la biblioteca della FFLCH, nella sezione dei libri d'italiano, una biblioteca dell'italianistica brasiliana. In tal modo qualunque ricercatore saprebbe dove cercare il materiale 
bibliografico e dove inviare una copia della propria produzione con la ragionevole certezza che in futuro sarà reperibile e letta. Ovviamente, se è difficile conoscere $\mathrm{e}$ reperire in Brasile ciò che l'italianistica brasiliana produce, a maggior ragione è da attendersi che questa produzione, salvo alcune eccezioni, non venga conosciura e letta in Italia. Eppure esiste una sede, la "Rivista Italiana di Dialettologia", che si sforza di segnalare periodicamente tutta la produzione che interessi la linguistica italiana fuori d'Italia. Nonostante la dedizione di chi cura questa sezione della rivista, non sempre però è possibile dar conto della produzione brasiliana, per la difficoltà dovura al reperimento delle informazioni necessarie ${ }^{2}$.

Fra le difficoltà che gli studiosi brasiliani incontrano, non ultima vi è la difficoltà di reperire le principali pubblicazioni italiane, siano esse libri o riviste. Le istituzioni, sia quelle universitarie sia quelle che rappresentano il governo italiano, non posseggono né ampie biblioteche ${ }^{3}$ né fondi sufficienti a formarle. Tuttavia una certa razionalizzazione è possibile, magari creando una banca dati di ciò che è disponibile nelle biblioteche delle varie sedi, utilizzando il prestito interbibliotecario e segnalando periodicamente le nuove ordinazioni. Anzi, proprio la grande difficoltà di comprare materiale italiano così importante per la comunità dovrebbe indurre a uno sforzo per mettere a disposizione di tutti ciò che arriva frammentato in varie sedi. La stessa difficoltà economica può in parte essere ridotta con una maggiore organizzazione che, come si dirà, permetterebbe il ricorso alle forme di finanziamento della ricerca messe a disposizione dalle agenzie di finanziamento.

Le difficoltà di comunicazione, se sono già evidenti fra coloro che aderiscono al ristretto ambito dell'italianistica brasiliana e ancor più fra costoro e l'Italia, diventano quasi assolute se si considera la precarietà dei rapporti fra gli italianisti e la più ampia comunità delle singole discipline che essa abbraccia. Si pensi alla scarsa partecipazione di chi lavora su temi di linguistica italiana (ma si potrebbe fare l'esempio della

2. La sezione è curata da Camilla Betroni. Per il Brasile si possono consultare le recensioni pubblicate nel numero XXIX (2005), per verificare quanto limitato sia l'accesso reale alle informazioni in merito.

3. Unica parziale eccezione è la biblioteca dell' Istituto di Cultura di S. Paulo. 
letreratura italiana o di altre discipline legate alla cultura italiana) col più ampio movimento dei linguisti brasiliani. Il rischio è ancora una volta l'isolamento, da una parte, a causa della specificità del tema di interesse, rispetto alla realtà locale, $\mathrm{e}$ dall'altra, a causa della specificità territoriale, rispetto all' Italia. Per evitarlo sarebbe perciò importante incoraggiare la partecipazione alle associazioni e agli eventi che raccolgono le singole discipline in Brasile. A questo scopo si segnala che il prossimo congresso della Associą̧ão Brasileira de Lingüistica (ABRALIN) si terrà nei primi mesi del 2007 a Belo Horizonte. Questo, come altri eventi analoghi per discipline diverse, è un'occasione preziosa per i linguisti italiani per farsi conoscere e far conoscere gli studi italianistici nel settore.

\section{1 rapporti con l'Italia}

Solo da poco e in pochissime occasioni mi pare che si siano stretri rapporti stabili di collaborazione e di ricerca effettivi tra l'italianistica brasiliana e quella italiana ${ }^{4}$. Normalmente gli accordi tra istituzioni universitarie iraliane e brasiliane si limitano di fatto allo scambio di studenti o ai corsi di formazione o di aggiornamento per i docenti, e non riguardano se non marginalmente la ricerca. Questo genere di collaborazione è tutcavia indispensabile per varie ragioni: innanzi tutto perché solo alimentandosi dei rapporti con l'Italia gli italianisti all'estero possono evitare l'isolamento disciplinare, con le inevitabili e ovvie conseguenze negative; in secondo luogo perché è da questi rapporti di collaborazione che nasce uno scambio proficuo fra le competenze, inevitabilmente diverse, di chi risiede in Italia e di chi risiede in realtà culturalmente altre; in terzo luogo perché ciò permetre agli studenti brasiliani di recarsi in Italia e automaticamente di inserirsi in progetti già struttura-

4. Personalmente conosco solo il caso della collaborazione fra gli italianisti della USP e l'Università di Trieste, rappresentata dal gruppo che fa capo a Elvio Guagnini, per le ricerche sul tema della letreratura di viaggio. ma sembra che varie sedi sciano tenrando di dare ai vari accordi già esistenti un impulso anche sul versante della ricerca. 
ti, di cui magari già fanno parte, e con docenti conosciuti e disponibili; in quarto luogo perché l'accademia italiana dispone sia di una tradizione più consolidata di quella brasiliana (indipendentemente dall'ambito disciplinare) sia di disponibilità finanziarie maggiori: tramite accordi con gruppi di ricerca italiana sarebbe infatti possibile accedere ai finanziamenti che l'Italia e l'Unione Europea offrono a progetti di collaborazione con l'America Latina; infine perché da queste collaborazioni privilegiate e costanti possono nascere corsi nuovi che riducano la distanza fra l'offerta di cui può godere uno studente in Italia e in Brasile.

E diventato ormai quasi un luogo comune sostenere che l'Italia non possiede una politica culturale, che non sostiene la diffusione della propria cultura o l'insegnamento della propria lingua fuori dai suoi confini come al contrario fanno gli altri grandi paesi occidentali come gli Stati Uniti, l'Inghilterra, la Germania, la Spagna, per non parlare della grande tradizione francese. Se ciò è senz'altro in parte vero, tuttavia è anche vero che le possibilità che esistono non vengono sfruttate adeguatamente. Ogni anno infatti il governo italiano, alcune istituzioni regionali e alcune università mettono a disposizione dei singoli o delle università straniere dove vi sia un insegnamento di italiano borse o fondi per vari obiettivi: viaggi di studio per studenti o docenti, acquisto di libri, contrattazione di lettori remporanei in loco, borse per studenti o per tesi di master o dottorato, fondi per organizzare manifestazioni o convegni, finanziamenti per corsi di aggiornamento per docenti. Concorrere a questi fondi presenta tuttavia alcune difficoltà: in primo luogo, ottenere le informazioni esatte sulle possibilità esistenti; in secondo luogo, redigere la domanda secondo le modalità gradite al ministero. Per queste ragioni è difficile che i docenti o gli studenti accedano direttamente e senza aiuti a tali fondi, ed è cruciale la partecipazione dei direttori didattici. Questa figura professionale, presente presso tutte le sedi consolari italiane in Brasile, conosce i canali per accedere ai fondi e può informare i docenti e aiutare a redigere le domande. Ne consegue che la collaborazione fra docenti e direttori didattici è cruciale.

Le istituzioni consolari possono, se lo desiderano, intraprendere in proprio varie iniziative di appoggio alle attività di ricerca delle università. Anche qui è ovviamente 
necessario che i docenti e le autorità consolari collaborino, e di nuovo appare cruciale il ruolo mediatore dei direttori didattici. Un esperimento in tal senso è in atto a Belo Horizonte, dove, grazie al sostegno della direttrice didattica e a una collaborazione a tre fra università, Fondazione Torino e Consolato, si sta tentando di dar vita a un ampio progetto di sostegno alla cultura italiana che sia di natura stabile e permetta alle varie entità interessate di ottenere fondi. I primi passi del progetto sono stati, da una parte, la richiesta di fondi al Ministero per poter contrattare localmente un lettore, per realizzare un breve convegno sui rapporti fra il Brasile e l'italiano, invitando relatori dall'Italia e da altre località brasiliane, e per invitare alcuni relatori italiani a partecipare al prossimo congresso della ABRALIN, che si svolgerà appunto a Belo Horizonte a inizio 2007; dall'altra, la realizzazione del primo volume di un repertorio che miri a raccogliere turti i dati interessanti per lo studio dei rapporti fra l'Italia, l'italiano e il Brasile nella circoscrizione consolare, indipendentemente dall'ambito disciplinare (storia, linguistica, arte, letteratura, economia, diritto, ecc.).

Al di là del successo o meno dell'iniziativa, essa si segnala per almeno tre aspetti importanti: il primo, e più significativo, è la collaborazione autentica fra i principali attori interessati alla crescita degli studi italianistici nel territorio, vale a dire le autorità che rappresentano il governo italiano, la Fondazione Torino, la cui missione biculturale fa della scuola il principale veicolo di italianità nella comunità, e i docenti di italiano o che lavorano su argomenti italiani presso l'Università Federale di Minas Gerais. Si tratta di una novità per questa circoscrizione consolare, ma anche altrove le esperienze di collaborazione sono state finora decisamente al di sotto delle potenzialità dei vari attori. Va da sé che, laddove esistono, anche altri attori dovrebbero essere coinvolti, primi fra tutti gli Istituti di Cultura. Il secondo aspetto è la decisione di dar vita a uno strumento fondamentale per lo sviluppo delle ricerche, vale a dire un repertorio; ciò permetterà infatti di mettere assieme le nocizie già esistenti e di definire, oltre a quanto già si è fatto, ciò che si può fare in futuro; la presenza di un repertorio permetterà ai futuri ricercatori di identificare $e$ reperire gli oggetti di ricerca e la bibliografia in maniera sistematica, e quindi ren- 
derà possibili programmi di ricerca non frammentari o casuali. Il terzo, infine, è il tentativo di rafforzare una comunicazione ingiustificatamente carente fra la realtà brasiliana di un determinato settore disciplinare, la linguistica, e l'equivalente settore italiano, magari con una comunicazione attiva tra ABPI e alcune delle associazioni disciplinari italiane, come la Società di Linguistica Italiana (SLI), l'Associazione per la Storia della Lingua Italiana (ASLI), l'Associazione Italiana di Linguistica Applicata (AitLA) o l'Associazione degli Italianisti (ADI)

Negli ultimi anni sono sorte in Italia alcune iniziative telematiche che interessano particolarmente gli studenti, ma anche gli studiosi, delle discipline italianistiche. La più importante è quella del consorzio ICoN (Italian Culture on the Net) ${ }^{6}$, un consorzio di più di venti università che offre corsi di italiano per stranieri e una laurea in Lettere riconosciuta dall'intero consorzio. Nel sito, a pagamento, si può trovare una ricchissima messe di moduli, prodotti da specialisti sui singoli argomenti, sulle varie discipline di interesse storico-letterario, dalla linguistica alla letteratura, alla storia, alla filosofia, allo spettacolo, alla geografia e alla antichistica; essi costituiscono una biblioteca completa sui vari aspetti della cultura italiana. Precedente a ICoN

5. Nacuralmente sarebbe importante ricevere i volumi che queste associazioni pubblicano periodicamente, in particolare la SLI e l'AIrLA. Analogamente sarebbe di grande utilità che la comunità brasiliana avesse accesso alle principali riviste che si pubblicano in Italia e ai principali repertori disciplinari, come la Storia d'Italia della Einaudi, la Storia della lingua italiana curata da L. Serianni e P. Trifone per la Einaudi, l'Italiano nelle Regioni curato da F. Bruni per la UTET, La Storia della lingua italiana del Mulino curata da F. Bruni, La Grande Grammatica di consultazione curata da L. Renzi, G. Salvi e A. Cardinaletri per Il Mulino, la Letterarura italiana curata da A. Asor Rosa per l'Einaudi, il Lexikon der romanistischen Linguistik curato per Niemeyer da G. Holrus, M. Merzeltin e C. Schmitr, o i repertori su CD come la Letteratura Italiana Zanichelli (L1Z), curata da Pasquale Stoppelli, che contiene il testo integrale di 1000 opere della letreratura italiana ed è dotata di un motore di ricerca urilissimo, ecc. Naturalmente la rete permette oggi di utilizzare diretramente molti strumenti utili, sia per la ricerca sia per la didarrica. Mi limito solo a citare la grande messe di mareriale disponibile grazie al progetro CIBIT (Centro Interbibliotecario Italiano Telematico) hrtp://cibit.humnet.unipi.it e, tra i vari corpora di italiano parlato, i] LIP (Lessico dell' Italiano Parlato) consultabile al sito hrtp://languageserver. uni-graz.at/badip/badip/home.php

6. Per la quale si veda il sito htep://www.iralicon.it 
è la produzione del progetto Italica della $\mathrm{RAI}^{7}$ Infine merita di essere menzionato il master ITALS organizzato dall'Università di Venezia ${ }^{8}$. Esso si svolge a distanza, con una fase finale in praesentia.

3 La pos-graduação c le agenzic di finanziamento

In alcune sedi universitarie, in primis São Paulo e Rio de Janeiro, esistono programmi di Pós-Graduação esclusivamente destinati agli studi italianistici. Tuttavia questo non è l'unico modello possibile e talvolta, considerato il numero inevitabilmente limitato dei docenti di italiano in una singola istituzione universitaria, può non essere il modello migliore per i nostri studi. In altre sedi, come accade ad esempio nella UFMG o nella UFSC, esiste la possibilità di studiare argomenti italiani all'interno di programmi più ampi (letteratura, linguistica, traduzione, storia, ecc.) sotto la guida di un italianista specializzato nella disciplina del programma. In sintesi, a casi in cui è stato possibile montare programmi generali di italianistica, si alternano casi in cui singoli italianisti sono inseriti in programmi disciplinari specifici ma trasversali per quanto riguarda la cultura o la lingua di studio.

Quale che sia comunque il modello scelto (e la scelta spesso dipende dalla struttura dell'istituzione e non dalla volontà del docente), sarebbe importante che le strategie di ricerca delle singole sedi eleggessero uno o pochi ambiti di lavoro privilegiato che possano configurarsi in progetri del programma e non solo del singolo. In tal modo si potrebbe nel tempo accumulare un'esperienza e una ricchezza di lavori con almeno due effetti positivi: la creazione di competenze forti su un settore di ricerca, con la conseguente possibilità di attrarre studenti e docenti interessati anche se non residenti nella singola sede, e la creazione di progetti forti, in grado di attrarre finanziamenti e suscitare visibilità fuori dalla dimensione locale. Infatti è noto che l'orientamento delle agenzie di finanziamento, come il CNPq o la
7. hetp://www.italica.org
8. hetp://www.itals.it 
CAPES, tende sempre più a privilegiare gruppi di ricerca ampi e che coinvolgono più sedi universitarie, perché esse danno maggiori garanzie di portare a termine progetti di dimensioni significative. Una strutturazione del genere potrebbe tra l'altro permettere seminari periodici che integrino le competenze di vari settori disciplinari e di varie sedi, alimentando i contatti, gli scambi e le collaborazioni. Insomma, la Pós-Graduação è un'occasione per creare col tempo dei veri e propri centri di ricerca su alcuni grandi temi, evitando la frammentazione di piccole ricerche isolate e distribuite su tutto il territorio, che spesso creano doppioni e che comunque non posseggono sufficienti forza e visibilità. Ciò non impedirebbe, ovviamente, che i singoli ricercatori continuino a coltivare i propri interessi, ma permetterebbe a costoro di inserirsi in ambiti strutturati, anche se la sede guida non è quella in cui lavorano.

Sopra si è detto della carenza dei rapporti fra il mondo accademico brasiliano e quello italiano. È noto infatti che gli studenti di dottorato in Brasile, indipendentemente dal fatto che lavorino su una determinata lingua, cercano, quando possono, la possibilità di recarsi per un anno o anche più fuori del proprio paese per formarsi alle più importanti scuole dei principali paesi occidentali: se gli Stari Uniti sono la meta principale di queste esperienze, tra i nomi dei paesi più richiesti compaiono anche quelli della Francia, dell'Inghilterra e perfino della Spagna, ma non quello dell'Italia, con l'unica parziale eccezione degli studi classici (caso nel quale il contrario sarebbe davvero impensabile). Ciò è vero per la linguistica, ma anche per altre discipline in cui l'Italia tradizionalmente contribuisce in maniera non inferiore ai paesi citati, come la letteratura, la storia, la filosofia. La ragione di ciò credo debba cercarsi, almeno in parte, nell'ignoranza da parte della realtà brasiliana di quanto si produca in Italia, anche se indubbiamente una quota di responsabilità andrà attribuita alla chiusura dell'ambiente universitario italiano, che offre poche opportunità a chi viene da fuori. Ciò non toglie che un italianista, indipendentemente dal settore disciplinare, possa e debba stimolare i rapporti fra la comunità scientifica del paese in cui vive e quella italiana, in modo che l'Italia e l'italiano non siano considerati solo il settore di pochi appassionari, ma in generale un veicolo di 
accesso a opportunità più qualificanti, sia per quanto riguarda la formazione, sia per conoscere una produzione scientifica più che competitiva con quella degli altri paesi citati ma non altrettanto nota qui in Brasile?

4 Cli argomenti di ricerca: guardare all'Italia guardando al Brasile

A mio parere la funzione di una comunirà di italianisti in Brasile, e in generale all'estero, è duplice: da una parte promuovere a tutri i livelli, dall'insegnamento alla ricerca, alle più svariate manifestazioni culturali, l'interesse per la cultura prodotta dall'Italia e in Italia; dall'altra, più specificamente, contribuire all'identificazione delle forme che l'italianità assume nel paese straniero. Mentre nel primo caso, salvo pochi casi eccezionali, si è faralmente costrerri ad agire di riflesso rispetto a quanto si fa in Italia, nel secondo l'italianista che risiede all'estero può giocare la parte del protagonista. È senz'altro più difficile, oltre che meno necessario, l'apporto di un italianista che risiede in Brasile allo studio di autori o fenomeni o forme culturali che sono nate e hanno avuto o hanno il loro corso nella penisola; è evidente che chi risiede in Italia possiede, a questo scopo, strumenti, contatti e competenze che inevitabilmente sono difficili da colmare per chi non vive quotidianamente a contatto con essi. Al contrario, a un italianista che risiede all'estero, e tanto più a chi risiede in un paese in cui la presenza italiana è storicamente così forte come il

9. Desidero qui citare due lavori recenti che meriterebbero la massima atrenzione da parte della comunità linguistica internazionale e quindi anche da parte di quella brasiliana e che un linguista italiano residente all'estero dovrebbe conoscere e far conoscere ai colleghi non italianisti: E. Cresti, Corpus di italiano parlato, Firenze, Accademia della Crusca, 2000, 2 voll.; E. Cresti e M. Moneglia (a c. di), C-ORAL-ROM. Integrated Reference Corpora for Spoken Romance Languages, Amsterdam, John Benjamins, 2005, com DVD. Non si tratta solo di un corpus dell'italiano e delle altre principali lingue romanze (francese, spagnolo e portoghese), ma di una reoria che integra e parametrizza l'analisi intonativa linguistica e pragmatica attraverso la corrispondenza fra enunciato e atto linguistico sulla base di elementi misurabili grazie all'analisi della curva intonativa. La teoria e il DVD forniscono quindi uno strumento eccezionale sia per la ricerca sia per la didatrica ad alco livello, non solo per l'italiano ma per la linguistica romanza e la linguistica tout court. Sulle iniziative del gruppo di ricerca si veda anche il sito http://lablita.dit. unifi.it. 
Brasile, è più agevole di quanto non sia per chi risiede in Italia studiare le forme in cui l'Italia ha stretto contatti con la realtà locale. Costoro infatti verosimilmente, se da una parte risentono della distanza dal centro di irradiazione della cultura italiana, dall'altro hanno l'opportunità di unire alle competenze dell'italianista quelle relative alla realtà e alla lingua locali, producendo quindi profili intellettualmente originali e capaci di riconoscere e interpretare la specifica realtà di contatto. Tali profili sono, a mio parere, una risorsa non sufficientemente valorizzata in molte realtà straniere e senz'altro in quella brasiliana, e, se maggiormente stimolati, permetterebbero alla comunità una visibilità ben maggiore.

Pertanto ritengo che, senza dimenticare la prima delle due funzioni indicate sopra, lo studioso della cultura italiana in Brasile debba privilegiare argomenti di ricerca in cui può svolgere una funzione originale e contribuire da protagonista e con competenze maggiori alla conoscenza della cultura italiana. Mentre infatti la comunità degli studiosi italiani presumibilmente non si aspetta che arrivi dal Brasile lo studio di un volgarizzamento toscano inedito o l'indagine storica degli archivi borbonici o l'analisi delle tradizioni popolari molisane o ancora la sistematizzazione dei dialetti friulani, si aspetterà invece dai colleghi brasiliani un contributo significativo sulla storia delle missioni italiane in territorio brasiliano o sui tanti fenomeni di contatto linguistico e culturale dove la presenza italiana nella società brasiliana è forte. Guardare all'Iralia e dialogare con essa significa quindi soprattutto dare un contributo originale e che difficilmente può essere portato da chi risiede in Italia. Naturalmente a questo fine non mancano gli argomenti di studio per l'italianistica brasiliana. Senza la pretesa di esaurirli, provo qui a indicare alcuni fra i principali filoni, cercando di andare oltre le competenze di una singola disciplina ${ }^{10}$.

10. Per quanto riguarda le intersezioni tra mondo italiano e mondo brasiliano lungo la storia $e$ in una prospettiva interdisciplinare è imprescindibile la conoscenza del volume promosso dalla Associazione Iralia-Brasilc Novamente retrovato. Il Brasile in Italia 1500-1995, Presidenza del Consiglio dei Ministri - Dipartimento per l'Informazione e l'Editoria, Roma, s.d. Il volume raccoglie saggi in un ampio raggio disciplinare che va dalla ctnografia alla storia culturale, dall'antropologia alla storia della scienza, dalla storia alla linguistica, dalla letreratura all'arte. 
Tra i tradizionali ambiti di indagine sulla presenza italiana in Brasile vi è senz'altro quello che riguarda l'emigrazione. Si tratta di uno dei temi più studiati, ma sempre attuale e ricco ancora di molti motivi di indagine ${ }^{11}$ Il tema è talmente vasto che si presta a molti approcci disciplinari, dalla storia economica o demografica, allo studio delle identità, alla sociolinguistica, all'antropologia, alla storia dell'arte, e ad altri approcci ancora. Se alcune realtà territoriali, come il sud e il sudest, sono state certamente toccate in modo più intenso dal fenomeno, non mancano casi significativi e poco noti in realtà territoriali diverse. L'argomento infatti, se affrontato non in termini quantitativi, può illuminare numerosi casi isolati ma importanti di presenza italiana nell'interno o nel nord del paese. Per una valutazione più approfondita del fenomeno e di quanto ancora resta da fare per la ricerca, è utilissima la collaborazione interdisciplinare con gli storici e una conoscenza degli archivi. Una indagine sistematica degli archivi potrebbe anzi costituire argomento di molte tesi di master o dottorato, nella considerazione del fatto che la creazione di un repertorio, per quanto limitato, significa sempre illuminare un frammento di storia culturale e creare l'opportunità per numerosissime ricerche che approfondiscano poi i singoli oggetri individuati dal repertorio.

Desidero insistere un po' su quest'ultimo aspetto, perché mi pare tanto importante quanto trascurato. Le possibilità di ricerca relative a un testo, a una figura o a un centro culturale dipendono ovviamente dalla conoscenza dell'esistenza del

11. La bibliografia sul tema è molto ricca. Mi limito qui a segnalare alcuni fra i tanti titoli significativi, dai quali può essere ricavata gran parte della produzione sull'argomento: G. Rosoli (a c. di), Un secolo di emigrazione italiana (1876-1976), Centro Studi Emigrazione, Roma, 1978; Angelo Trento, Là dovê La raccolta del caffe. Lemigrazione italiana in Brasile, Padova, Antenore, 1984; idem, Do outro lado do Atlantico. Um século de imigrą̧äo italiana no Brasil, Sāo Paulo, Nobel, 1989; alcuni dei lavori contenuti nei testi seguenti: Boris Fausto (a c. di), Fazer a América, São Paulo, Edusp, 1999; P Bevilacqua, A. De Clementi, E. Franzina, Storia dellemigrazione italiana, Roma, Donzelli, 2002, 2 voll.; Luis A. De Boni (a c. di), A presenfa italiana no Brasil, Ediçōes EST-Fondazione Giovanni Agnelli, Porto Alegre-Torino, 1987-1996. 3 voll.; G. Tassello, M. Vedovelli, Scuola. lingua e cultura nell'emigrazione italiana all'estero. Bibliografia generale (1970-1995), Roma, Centro Studi Emigrazione, 1996. 
testo o della figura o del centro culturale che si vuole studiare. Se ciò è facile per testi, figure e centri recenti o che hanno ricevuto una notevole risonanza editoriale o altre forme di pubblicità, non è cosl invece per un'ampia messe di testi o di personalità o per filoni culturali che sono rimasti inediti o che hanno goduto di una vita effimera e sono poi stati risucchiati dall'anonimato. Eppure essi hanno agito e spesso meritano di essere recuperati, talvolta per il loro valore intrinseco, altre perché illuminano un'epoca o un'area culturale. Questo genere di oggetti deve quindi prima essere portato alla luce e solo dopo può essere studiato. Da ciò l'utilità estrema della produzione di repertori. Entrare in un archivio o in una biblioteca importante e individuare a tappeto i materiali che possono interessare un approccio disciplinare, lungi dall'essere un lavoro poco stimolante e meccanico, è un operazione di grandissima utilità per almeno due ragioni: la prima è che un lavoro del genere individua una costellazione (di testi, di autori, di percorsi) e costituisce quindi di per sé un'operazione di grande importanza storico-culturale; non si tratta infatti solo di elencare ciò che si trova, ma anche di capire come e per quali tramiti i materiali sono giunti nel luogo ove si trovano, di dare uno sguardo sommario a ogni unità e fornire una prima pista per un'analisi più approfondita, di strutturare il materiale in sottogruppi omogenei per interesse, ruolo storico, autori. In secondo luogo, uno strumento del genere permette a molti altri studiosi di realizzare ricerche nuove che approfondiscano uno dei singoli elementi portati alla luce. In conclusione, studiare un archivio, o anche una parte di esso, deve di per sé essere considerato un lavoro ampiamente utile e meritorio, senza il quale si chiudono le porte alla comprensione di tutto ciò che non è immediatamente noto e percepibile.

Una fonte interessante, sia per gli studi storici sia per chi ha interessi antropologici o linguistici, è costituita dai numerosi libri di viaggio, che percorrono cinque secoli di storia, dal momento che gli italiani sono tra i viaggiatori più noti sin dalla scoperta del nuovo continente ${ }^{12}$.

12. Si veda per questo Associazione Italia-Brasile (a c. di), cit. e, tra i contributi recenti più interessanti, il lavoro di C. Palma dos Santos, in "Revista de Italianistica”, IX, 2004, pp. 83-98. 
Il Brasile, come è noto, è un paese di grandissimo interesse per gli studi antropologici, etnografici ed etnolinguistici e ciò è vero anche per indagare come la religiosità o la quotidianità e gli usi delle componenti etniche italiane siano stati reinterpretati nella società brasiliana. Non è difficile immaginare interessanti ambiti di indagine come il peso assunto da culti decisamente periferici nel paese di origine ${ }^{13}$ e la loro rilettura in loco, oppure la reinterpretazione delle tradizioni gastronomiche a cavallo fra memoria e contaminazione con gli usi e i prodotri del paese di adozione, o ancora la presenza di intercalari, parole o usi linguistici italiani o più spesso dialettali in molte comunità, quando non addirittura il riflesso della enorme componente italiana nella fonetica del portoghese di regioni come S. Paulo, oppure lo studio di elementi del costume e della cultura di massa come le telenovelas in cui compaiono personaggi italiani o che addirittura ripropongono la saga dell'emigrazione, e ancora i segni evidenti nelle insegne dei negozi e nella quotidianità dei brasiliani dell'enorme prestigio di cui gode l'immagine dell'Italia oggi nel paese.

Quest'ultimo esempio ci porta al più ampio argomento della ricezione dell'italianità in un paese così variegato e ampio come il Brasile. Si tratta di una prospetriva feconda per tutti gli interessi disciplinari, dalla storia, all'antropologia, alla letteratura, all'arte, alla lingua. Infatti un conto è la cultura italiana così come è nata e si è evoluta nella madrepatria, e un conto ben diverso è ciò che questa cultura ha prodotto a contatto con altre culture e più o meno isolata dal contesto italiano. Larte e l'architettura italiana hanno condizionato urbanisticamente e culturalmente interi quartieri di città brasiliane ${ }^{14}$, così come il sistema fondiario del sud del paese risente

13. Si pensi ad csempio al culto della Madonna Achiropita nella comunità italiana di S. Paulo.

14. Si pensi al caso della costruzione di Belo Horizonte, avvenuta ad opera di maestranze italiane, su cui si veda Ambasciata d'Italia, Presenza italiane in Brasile, São Paulo. Istituto di Cultura di San Paolo-I.C.I.B. Istituro di Culcura Italo-Brasileiro, s.d., pp. 11 sgg., ma anche a tante ville di S. Paulo. Su S. Paolo si veda Bruno Giovannetri (a c. di), Arquitetura Italiana em São Paulo. São Paulo, 1994 e, dello stesso autore, Artistas Italianos nas Praças de S. Paulo, Empresa das Artes, 1992. 
della struttura proprietaria del nordest italiano. La letteratura classica e quella italiana hanno influenzato generazioni di scrittori brasiliani, a cominciare da Machado de Assis, e continuano a influenzarla. Spesso a influenzarla non è il prestigio letterario della cultura italiana, ma è direttamente il riferimento all'idea o a una idea dell'Italia che ispira la narrazione ${ }^{15}$. A questo proposito, sarebbe interessante avere un quadro storico delle traduzioni in portoghese delle opere italiane. Un'indagine di questo tipo contribuirebbe a conoscere quali sono le linee della cultura italiana che hanno agito nella formazione degli intellettuali brasiliani ${ }^{16}$. Analogamente sarebbe interessante vedere cosa oggi si pubblica di italiano in lingua portoghese e quali sono gli indici di consumo; ciò permetrerebbe di cogliere un importante canale che veicola l'immagine dell'Italia in Brasile ${ }^{17}$ A questo argomento, chi possiede interessi letterari può aggiungere il tema, particolarmente in voga, della letteratura di emigrazione ${ }^{18}$, sia, soprattutto storicamente, prodotra da italiani in Brasile, sia, forse con maggiore ricchezza di opportunità oggi, prodotta da brasiliani in Italia.

15. Per citare solo due esempi recenti, si pensi all'idea della mafia e del mondo siciliano alla base di un romanzo come Honra ou vendetta di Silvio Lancellotri c all'idea del legame fra Italia c tradizione anarchica in Um amor anarquista di Miguel Sanches Neto.

16. Si pensi, per tempi recenti o non lontani al prestigio di cui godono o hanno goduto in Brasile autori come Gramsci, Pasolini, Bobbio o Eco, per non parlare dell'enorme fortuna di Machiavelli. La parricolare sensibilità del paese per questi autori e non per altri di importanza analoga o maggiore deve trovare una spiegazione nei meccanismi della ricezione.

17. La ricerca invece si è già interessata alle pubblicazioni in lingua italiana all'estero; per il Brasile si trovano notizie nei resti citati che hanno indagato il fenomeno migratorio. Si veda comunque Assocamerestero, $\mathrm{CCIE}$, Unioncamere e MAE - Direzione Generale Emigrazione e Affari Sociali (a c. di), I media della diaspora. Giornali, radio e selevisioni dell'talia fuori d'Italia, Presidenza del Consiglio dei Ministri, Dipartimento per l'Informazione e l'Editoria, Roma, 1994. Vale la pena di segnalare qui le iniziative dell'editrice Berlendis e Verrecchia che negli ultimi anni ha pubblicato numerose opere letterarie italiane.

18. J-J. Marchand (a c. di), La letteratura dell'emigrazione, Gli scrittori di lingua italiana nel mondo, Torino, Fondazione Giovanni Agnelli, 1991. 
Si può dire che non vi sia quasi ambito della cultura brasiliana che non risenta, direttamente o indirettamente di un contatto con la cultura italiana, sia nel senso che il prestigio, antico e moderno, dell'Italia e della sua cultura ha nutrito la vita brasiliana come quella di altri paesi, sia, a maggior ragione, perché i legami stretrissimi di larga parte della società brasiliana con l'Iralia hanno da più di un secolo riservato all'Italia un posto di rilievo fra i modelli culturali a cui guardare per la formazione dell'identità nazionale; un modello che è diventato parte così integrante della società da non essere più vincolato a una origine etnica delle singole persone.

Non vi è dubbio che le tracce lasciate dall'Italia in quasi un secolo e mezzo sulla società brasiliana siano talmente forti, sia sotto il profilo quantitativo sia sotto quello qualitativo, da averne condizionato profondamente la formazione di un'identità nazionale originale. Il Brasile è senz'altro lusofono, nonostante siano più che evidenti gli effetri del contatto linguistico indigeno, africano e con le varie realtà di immigrazione (prima fra tutte quella italiana), ma non può dirsi culturalmente portoghese più di quanto non sia africano o italiano. Per un italianista ciò è una enorme opportunità: lavorare avendo sullo sfondo la ricerca del contributo italiano alla formazione dell'identità brasiliana, cioè quanto di ciò che il Brasile è oggi è dovuto alla ricezione e alla rielaborazione originale di elementi di italianità, e come avviene il processo di tale rielaborazione ${ }^{19}$

Per tutti questi ambiti di indagine, e per moltissimi altri ancora, è indispensabile possedere sia una competenza profonda dal versante italiano sia una conoscenza intima della realtà brasiliana e della sua storia. E occuparsi di argomenti del genere significa illuminare importanti capitoli della storia culturale italiana fuori di Ita-

19. Su questo tema alcune rillessioni si possono trovare in T. Raso, Litaliano in Brasile oggi, in "Quaderni iberoamericani", 85-86, 1999. pp. 109-118 e natumelmente in Sérgio Buarque de Hollanda, $A$ contribuiçäo italiana para a formąão do Brasil, Florianópolis, NUT/NEIITA/UFSC, 2002. 
$\operatorname{lia}^{20}$. Per entrambe queste ragioni è ragionevole ritenere che questi siano gli ambiti naturali della ricerca per un italianista che vive in Brasile.

\section{La lingua}

Data la natura di questa rivista e del suo pubblico, e date le competenze di chi scrive, non si può non concludere questo breve intervento con uno sguardo più puntuale agli interessi linguistici ${ }^{21}$ Si è già accennato sopra ad alcuni ambiti di indagine sociolinguistico, come è il caso delle comunità venete del sud del paese. $\mathrm{Na}-$ turalmente esistono comunità interessanti anche in altre regioni, come testimoniano i lavori su Pedrinhas Paulista. Si tratta di ricerche che affrontano il problema del bilinguismo in parlanti non colti, con una conoscenza dell'italiano limitata, sia che si

20. Si tratta di una storia molto più ricca di quanto comunemente si pensi e ancora in buona misura da scrivere, come dimostrano le recenti scoperte sul cosiddetto Levant italian, su cui si veda F. Bruni, Lingua d'sltremare. Sulle tracce del "Levant Italian' in età preunitaria, in "lingua nostra", 60, 1999, pp. 65-79. A proposito dell'italiano fuori di Italia si vedano anche S. Vanvolsem, D. Vermandere, Y. D'Ulst, F. Musarra (a c. di), Litaliano oltre frontiera, atti del V convegno internazionale, Leuven-Firenze, Leuven University Press-Cesati, 2000, 2 voll; Società Dante Alighieri, Vivere italiano: il futuro della lingua, rapporto dell'indagine svolea in collaborazione con il CNEL, Roma, 1999; L. Renzi, M. Cortelazzo (a c. di), La linguistica italiana fuori d'Italia. Studi, Istituzioni, SLI, 38, Bulzoni, Roma, 1997; T. De Mauro, M. Vedovelli, La diffusione dell'italiano nel mondo e le vie dell'emigrazione. La prospettiva degli anni' '90, Roma, Centro Srudi Emigrazione, 1996; T. De Mauro, M. Vedovelli, M. Barni, L. Miraglia, Italiano 2000. Indagine sulle motivazioni dei pubblici dellitaliano L2 nel mondo, Roma, Ministero degli Affari Esteri, 2001; A. Giacalone Ramat, M. Vedovelli, Italiano lingua secondallingua straniera. Atti del XXVI congresso della Società di Linguistica Italiana, Roma, Bulzoni, 1993. Utile dal punto di vista storico, ma ormai invecchiaro nelle osservazioni in sincronia, è anche V. Lo Cascio (a c. di), Litaliano in America Latina. Congresso internazionale svoltosi a Buenos Aires, Firenze, Le Monnier, 1987.

21. Per uno sguardo sulla produzione nelle varie branche della linguistica italiana fino a pochi anni fa, si può consultare C. Lavinio (a c. di), La linguistica italiana alle soglie del 2000 (1987-1998), Bulzoni, Roma, 2002. dove il contributo di M. Vedovelli è specificamente dedicato all' Italiano come L2 (pp. 160-212) e fornisce un quadro dei principali problemi affrontati nell'ambito e una ricchissima bibliografia di ben 18 pagine. 
tratti di lavori di carattere storico su fonti scritte di emigranti di prima generazione, sia che si tratti di lavori sulla seconda o la terza generazione. Si tratta quindi di una prospettiva di studio sull'erosione linguistica e sui risultati del contatto che tocca inevitabilmente questioni di ordine sociale: un approccio che gode di metodologie consolidate e che è sempre molto interessante e ricco di possibilità ${ }^{22}$.

Chi scrive ha invece avviato alla fine del 2004 un progetto sull'erosione dell'italiano colto in contatto con il portoghese brasiliano. Al progetto partecipano docenti e studenti di più sedi universitarie. In realtà l'idea di studiare il fenomeno dell'erosione degli italiani colti è più antica ed è da attribuire a Loredana Caprara e al gruppo della USP ${ }^{23}$. Questa linea di ricerca ha obiettivi e sfrutta metodologie diversi dalla precedente, perché studia l'erosione da un punto di vista prettamente linguistico e sincronico; per essa, costituendo lo studio dell'erosione l'altra faccia della medaglia dell'acquisizione, sono di interesse primario anche i meccanismi di apprendimento,

22. Tra i molti studi sul sud del Brasile ricordo G. Meo Zilio (a c. di), Presenza, cultura, lingua e tradizioni dei veneti nel mondo. Parte I America Latina. Prime inchieste e documenti, Venezia, Giunta Regionale Veneto, 1987; G. Padoan (a c. di), Presenza, cultura, lingua e tradizioni dei Veneti nel mondo, II, Venezia, Regione Veneto, 1994; Florence Carboni, "Eppur si parlano". Étude diacranique d'un cas de contact linguistique dans le Ris Grande do Sul (Brésil), Passo Fundo, UPF, 2002; V. M. Frosi, C. Mioranza, Dialetos italianos, Caxias do Sul, EDUCS, 1983; E. Franzina, Merica! Merica! Emigrazione e colonizzazione nelle lettere dei contadini veneti e friulani in America Latina. 1876-1902, Verona, Cierre, 1992; U. Bernardi (a c. di), A catar fortuna. Storie venete d'Australia e del Brasile, Vicenza, Giunta Regionale del Veneto, Neri Pozza, 1994, dove compaiono due contributi linguistici sul Rio Grande do Sul di G. B. Pellegrini. Si vedano inoltre i vari contriburi di L. Corrà e E. Ursini, di cui cito solo Migranti romanzi in età moderna in G. Holtus, M. Merzeltin e C. Schmitr (a c. di), Lexikpn der romanistischen Linguistik, 7, Tubingen, Niemeyer, 1998, pp. 559-585. Per S. Paulo, lavorano sull'argomento Loredana Caprara e Giliola Maggio de Castro.

23. Si vedano in proposito l'intero numero 5 della "Revista de Icalianistica" e T. Raso, L'italiano parlato a San Paolo da madrelingua colti. Primi sondaggi e ipotesi di lavoro, in "Revista de Italianisrica", VIII, 2003, pp. 9-49. II progetto avviato a Belo Horizonte mira ad ampliare il corpus predisposto a S. Paulo e a inserirlo nel progetto CHILDES, su cui si vedano B. MacWhinney, Il Progetto CHILDES (edizione icaliana a cura di E. Pizzuto e U. Bortolini), Pisa, Edizioni del Cerro, 1995; U. Bortolini e E. Pizzuto (a c. di), Il Progetto CHILDES Italia, Pisa, Edizioni del Cerro, 1997; e il siro hrtp://childes.psy.cmu.edu 
tanto della L1 quanto della L2, e lo studio contrastivo delle due lingue di contatto 24 per poter cogliere i punti del sistema più esposti al fenomeno.

Entrambi gli approcci però toccano, sia pure in maniera e in proporzioni diverse, aspetti importanti dei fenomeni di contatto, sia sotto il profilo sociolinguistico, come i temi legati all'identità, al prestigio, all'origine socio-culturale e all'inserimento sociale, sia sotto il profilo testuale, come la commutazione di codice (il cosiddetto code-switching) e la scelta dei registri, sia sotto il profilo acquisizionale, come i meccanismi di perdita e di acquisizione linguistica ${ }^{25}$.

Lo studio dell'acquisizione dell'italiano come L2 occupa naturalmente un posto di rilievo di per sé. Si tratta di un tipo di studi oggi molto praticato, sia sotto il profilo squisitamente linguistico sia sotto quello glottodidattico. Quest'ultimo si concentra nell'individuazione delle tecniche che rendano più efficace l'apprendimento di una L2 e nella produzione di materiale indicato a questo obiettivo. Dato lo sviluppo recente della glottodidattica ${ }^{26}$ in Italia e data la forte contiguità fra glottodidattica e opportunità di contatti fra istituzioni italiane e università straniere, direi che quest'area è quella che oggi più facilmente e frequentemente consente scambi con le università italiane, in particolare quelle che dispongono di un forte centro glottodidattico, e opportunità di finanziamenti, fra cui Venezia, Roma, e naturalmente le Università per Stranieri di Perugia e Siena ${ }^{27}$ Non altrettanto diffuso, ma molto interessante, è lo studio dell'apprendimento in chiave acquisizionale. In Italia il centro principale di questi studi è a Pavia e il suo oggetto di indagine teorica è il meccanismo di apprendimento di una L2, con un forte accento sulla

24. Un esempio di analisi contrastiva a proposito dell'aspetto verbale del pretérito perfeito composto è l'arricolo di M. E. Verdaguer, in "Revista de Italianística", IX, 2004, pp. 185-201.

25. Su turti questi temi la bibliografia c̀ ampia e di facile reperimento anche in Brasile.

26. Cito qui alcuni dei manuali di glotrodidatrica pubblicari in Italia: A. Ciliberti, Manuale di glottodidattica, Firenze, La Nuova Italia, 1994; P. E. Balboni, Didattica dell'italiano a stranieri, Roma, Bonacci, 1994; P. Margurci, Comunicare in una lingua straniera. Dalla teoria alla pratica, Roma, Carocci, 2004.

27. Un'utile guida ai temi che riguardano l'insegnamento dell'italiano agli stranieri è M. Vcdovelli, Lïtaliano degli stranieri. Storia, attualità e prospetrive, Roma, Carocci, 2002. 
cosiddetta interlingua, cioè quei sistemi linguistici naturali e in equilibrio dinamico che costituiscono la competenza di un apprendente una L2 durante la fase di apprendimento e quindi senza coincidere con la L2 stessa ${ }^{28}$. Lo studio delle interlingue è di grande interesse teorico e in forte espansione. Uno degli oggetti di studio più stimolanti, quindi, anche per un linguista italiano che vive all'estero è proprio lo studio dell'acquisizione dell'italiano come L2 in quel contesto linguistico. A questo proposito il Brasile dispone non solo del materiale fornito dai vari apprendenti nei corsi universitari o in altri corsi, ma anche della situazione privilegiata rappresentata dalle due scuole bilingui e biculturali della Fondazione Torino a Belo Horizonte e della Eugenio Montale di S. Paulo.

In parte connesso a questa prospettiva di studio, sarebbe utilissimo uno sforzo per illuminare contrastivamente gli aspetti pragmatici e culturali del linguaggio. $\mathrm{E}$ noto che, al di là del diverso codice linguistico, i parlanti di due comunità nazionali come Italia e Brasile non condividono le strategie testuali. Spesso la comunicazione si inceppa o non funziona non per difficoltà imputabili al codice, cioè non per ragioni di ordine grammaticale o lessicale, ma per come le due culture codificano i singoli atti linguistici, cioè per ragioni di ordine pragmatico. Questo aspetto può essere ancora molto incrementato sia nella ricerca sia nell'insegnamento e viene sempre più richiamato nella teoria didattica ${ }^{29}$ Tuttavia, perché esso possa essere

28. Tra i manuali pubblicati in Italia sull'argomento segnalo due testi dovuti ai ricercatori del gruppo di Pavia: A. Giacalone Ramat (a c. di), Verso l'italiano. Percorsi e strategie di acquisizione, Roma, Carocci, 2003; M. Chini, Che cosê la linguistica acquisizionale, Roma, Carocci, 2005; e C. Betconi, Imparare unialtra lingua, Roma, Latera, 2001. Chi volesse consultare i dati del progetro di Pavia può farlo in C. Andorno (a c. di). Banca Dati Italiano L2. Progetto di Pavia, CD ROM, Università di Pavia, Dipartimento di Linguistica, 2001.

29. Si pensi all' importanza guida che esso assume nel Quadro comune europeo di riferimento per le lingue: apprendimento, insegnamento, valutazione. Il documento dे reperibile in inglese $\mathrm{e}$ in altre lingue nel sito htrp://culture. coeffr/lang, mentre la versione iraliana è uscita a stampa a cura di Franca Quartapelle e Daniela Berrocchi, Milano-Firenze, RCS Scuola-La Nuova Ialia, 2002. Un diffuso commento al Framewark in funzione di un modello glottodidatrico si trova in M. Vedovelli, Guida all'italiano per stranieri. La prospettiva del Quadro comune europeo per le lingue, Roma, Canocci, 2002. 
utilizzato in modo non impressionistico nell'insegnamento, mancano ancora studi appropriati $^{30}$

RESUMO: $O$ artigo avalia a situaçâo da italianistica brasileira sob o aspecto da pesquisa e oferece alguns motivos de reflexāo para ofuturo. Por um lado se avalia o aspecto organizacional dos estudos, da pesquisa e das trocas entre colegas e com a Itália. Por outro lado se dá alguma sugestāo de áreas de interesse prioritário para os estudos italianisticos no Brasil. O foco principal é sobre a necessidade de contribuir com estudos que aprofindem as áreas de contato entre Brasile Itália. Além disso, o artigo fornece as indicaçōes dos principais instrumentos bibliográficos para o italianista brasileiro.

PALAVRAS CHAVE: italiano; pesquisa; contato.

30. Per un primo approccio al problema in rapporto all'italiano e per una bibliografia, si veda P. E. Balboni, Parole comuni culture diverse. Guida alla comunicazione interculturale, Venezia, Marsilio, 1999. 